\title{
Relationship between Metabolic Syndrome and MTHFR Polymorphism in Colorectal Cancer
}

\author{
Bong Su Kang, Dae Ho Ahn, Nam Keun Kim', Jong Woo Kim \\ Departments of Surgery and ${ }^{1}$ Institute for Clinical Research, CHA Bundang Medical Center, CHA University School of Medicine, Seongnam, \\ Korea
}

Purpose: There have been studies on the relations between metabolic syndrome and colorectal cancer or on the relations between methylenetetrahydrofolate reductase (MTHFR) polymorphism and colorectal cancer, but reports on the relationship between metabolic syndrome, MTHFR polymorphism and colorectal cancer all together are rare. The aim of this study is to find the interrelation between metabolic syndrome and MTHFR polymorphism in colorectal cancer.

Methods: This study investigated 255 colorectal cancer patients (cancer group) who underwent surgery in our hospital from March 2003 to December 2008 and compared those patients to 488 healthy patients (control group). The diagnostic criterion for metabolic syndrome was based on the National Cholesterol Education Program-Adult Treatment Panel III (NCEP ATP III), and the MTHFR 677 polymorphism was analyzed.

Results: When colorectal cancer patients and patients in the control group were classified as MTHFR 677 subtypes, there was no difference between the two groups: CC 87 (34.1\%), CT 134 (52.6\%), and TT 34 (13.3\%) for the cancer group and CC 145 (32.4\%), CT 238 (53.1\%), and TT 65 (14.5\%) for the control group. Distributions of MTHFR 677C/T genotype and allele frequencies in the individuals with and without metabolic syndrome in the cancer group showed no differences. Moreover, we could find no differences in distributions of MTHFR 677C/T genotypes in the clinical and the biomedical variables of individuals with and without metabolic syndrome in the cancer group.

Conclusion: Our results show no relation between metabolic syndrome and MTHFR polymorphism in colorectal cancer. However, a further prospective study, based on a precise diagnostic criterion for metabolic syndrome, is needed.

Keywords: Metabolic syndrome; MTHFR; Colorectal neoplasms

\section{INTRODUCTION}

Recently, the incidence of colorectal cancer in Korea has been increasing sharply, and it now ranks second among digestive organ malignancies. This is thought to be related to the rapid change in the life style of Koreans during past few decades due to industrialization, increased amounts of high-calorie foods and fat, etc. [1]. Colorectal cancer seems to have complicated and diverse contrib-

Received: February 16, 2011 Accepted: April 18, 2011

Correspondence to: Jong Woo Kim, M.D.

Department of Surgery, CHA Bundang Medical Center, 351 Yatap-dong,

Bundang-gu, Seongnam 463-712, Korea

Tel: +82-31-780-5250, Fax: +82-31-780-5259

E-mail: kjw@cha.ac.kr

(c) 2011 The Korean Society of Coloproctology

This is an open-access article distributed under the terms of the Creative Commons Attribution NonCommercial License (http://creativecommons.org/licenses/by-nc/3.0) which permits unrestricted noncommercial use, distribution, and reproduction in any medium, provided the original work is properly cited. uting factors, including genetic tendency; environmental factors, such as dietary food, chemicals and pollution; and personal factors, such as reduced activity, obesity, hyperlipidemia, hyperinsulinemia, etc., which usually come together. In industrialized countries with aging societies, such risk factors have already been already identified. In certain patients, metabolic disorder might have a large influence in the development of colorectal cancer. In 1988, Reaven et al. [2] denominated those patients as insulin-resistant syndrome or Syndrome-X.

In 1998, the World Health Organization (WHO) defined the concept of disorders as metabolic syndrome and suggested guidelines for diagnosis. Soon after, several groups, such as the National Cholesterol Education Program-Adult Treatment Panel III (NCEP-ATP III) and the International Diabetes Foundation (IDF), have suggested modified guidelines for making an accurate diagnosis [3, 4]. In the past, metabolic syndrome was thought to be a risk factor related only with cardiovascular disease; however, it has also been considered to be directly and indirectly related to other dis- 
eases such as cancer [2, 3]. Sturmer et al. [5] studied 494 colorectal cancer patients in a population of 22,071 and reported obesity and diabetes as risk factors causing colorectal cancer. Nevertheless, they could not find any relationship with hypertension and hypercholesterolemia. Ju et al. [6], reported a domestic study with 558 adult patients, in which metabolic syndrome might have increased the incidence of colorectal polyps, particularly in males.

There are two major pathways of colorectal carcinogenesis, the chromosomal instability pathway and the microsatellite instability pathway, which are based on epigenetic alterations, such as hypermethylation or hypomethylation, of genes. Methylenetetrahydrofolate reductase (MTHFR) is one of the key enzymes in the process of folic acid metabolism, which is an important substrate for DNA synthesis. It has a major role in DNA methylation and has a close association with the synthesis of methionine. According to the polymorphism of the enzyme, the strength of the enzyme changes the reduction of methionine synthesis and DNA methylation. Based on several reports, this alteration seems to be associated with the carcinogenesis of the colorectum $[7,8]$.

Abnormal folic acid metabolism and hyperhomocysteinemia are known to increase the risk of coronary heart disease. Oron-Herman et al. [9] reported that hyperhomocysteinemia was an imminent factor in metabolic syndrome in a mouse model study with polymorphism of MTHFR. Ellingrod et al. [10] said that patients with the T allele in MTHFR polymorphism had a 3.7-fold increased risk of having metabolic syndrome. On the contrary, the CC wild genotype exhibited a positive relationship for the schizophrenic patients having psychiatric drugs.

So far, reports studying the relationship between metabolic syndrome and MTHFR polymorphism in colorectal cancer patients are rare, and for that reason, this study was aimed at investigating the mutual relation between MTHFR polymorphism and metabolic syndrome in colorectal cancer patients as another associated risk factor.

\section{METHODS}

\section{Subjects}

We conducted a retrospective study on 255 colorectal cancer patients who underwent surgery from March 2003 to December 2008. For the control group, 448 healthy adults without any disease, such as cancer or metabolic disease, who showed similar ages and gender distributions, were selected. The Institutional Review Board (IRB) of our Medical Center approved this genetic study in June 2009.

\section{Diagnosis of metabolic syndrome}

In this study, we adopted the definition of metabolic syndrome from NCEP-ATP III. Patients having more than two of the following factors were defined as having metabolic syndrome; obesity, body mass index (BMI) $\geq 25 \mathrm{~kg} / \mathrm{m}^{2}$; triglyceride, TG $\geq 150 \mathrm{mg} / \mathrm{dL}$; high density cholesterol, male $<40 \mathrm{mg} / \mathrm{dL}$ and female $<50 \mathrm{mg} / \mathrm{dL}$; fasting blood sugar, FBS $\geq 110 \mathrm{mg} / \mathrm{dL}$; and blood pressure, systolic $\mathrm{P} \geq 130 \mathrm{~mm} \mathrm{Hg}$ or diastolic $\mathrm{P} \geq 85 \mathrm{~mm} \mathrm{Hg}$. We used the BMI, instead of abdominal circumference, as one of the diagnostic criteria.

\section{Analysis of MTHFR polymorphism}

Specimens were taken from leukocyte or formalin-fixed paraffinembedded specimens by using phenol/chloroform extraction methods, and the quality was adequate. The MTHFR genotype was examined using the PCR restriction fragment length polymorphism (RFLP) method. The DNA fragment containing MTHFR 677 C $>\mathrm{T}$ (Ala to Val) was amplified with a GeneAmp PCR machine (Perkin Elmer 2400, Foster City, CA, USA). DNA was replicated with human genomic DNA $200 \mathrm{ng}, 1.5-\mathrm{mM} \mathrm{MgCl}_{2}, 0.2-\mathrm{M}$ deoxynucleotide triphosphate, 1 unit Taq Polymerase (TaKaRa, Madison, WI, USA), and primer 100 pmol. The DNA sequence of the forward primer was 5'-GCACTTGAAGAGAAGGTGTC-3', and that of the reverse primer was $5^{\prime}$-AGGACGGTGCGGTGAGAGTG-3'. The PCR was set at 35 cycles in $94^{\circ} \mathrm{C}$ for 30 seconds, $51^{\circ} \mathrm{C}$ for 30 seconds and $72^{\circ} \mathrm{C}$ for 30 seconds after a period of denaturation for 5 minutes at $94^{\circ} \mathrm{C}$. The terminal portion of the DNA was elongated at $72^{\circ} \mathrm{C}$ for 5 minutes. The products of PCR were precipitated in ethanol, disintegrated by Taq I restriction enzyme, and finally treated by using electrophoresis. The variant allele in the 677 $\mathrm{C} \rightarrow \mathrm{T}$ polymorphism causes cytosine to be replaced with thymine in the final peptides. The TT variant type shows two bands, $173 \mathrm{bp}$ and $30 \mathrm{bp}$, the CT heterozygote type shows three bands, $203 \mathrm{bp}$, $173 \mathrm{bp}$ and $30 \mathrm{bp}$, and the CC wild type shows a single band, 203 bp, on electrophoresis. As a qualifying control, DNA from the lymphoblastoid cells with the three types of polymorphisms was used.

\section{Data analysis}

All calculations were done with Graphpad prism 4.0 (Graphpad Software Inc., San Diego, CA, USA), and categorical data were analyzed using the Fisher's exact test and the t-test. The MedCalc ver. 10.4.0.0 (MedCalc Software Inc., Mariakerke, Belgium) was used for the ANOVA test and the chi-square test. Statistical significance was accepted at $\mathrm{P} \leq 0.05$.

\section{RESULTS}

Among the 255 cancer patients, the metabolic syndrome group included 110 patients (43\%) and the non-metabolic syndrome group included 145 patients (57\%). A comparison of these two groups based on the diagnostic criteria of metabolic syndrome, including BMI, triglyceride, HDL cholesterol, fasting blood sugar, and systolic and diastolic blood pressure, showed statistically significant differences (Table 1).

MTHFR 677 polymorphism in cancer patients was distributed as follows: 87 cases (34.1\%) of CC type, 134 cases (52.6\%) of CT type, and 34 cases (13.3\%) of TT type. The control group showed a distribution similar to that of the cancer group with 145 cases (32.4\%) of CC type, 238 cases (53.1\%) of CT type and 65 cases (14.5\%) of 
TT type (Table 2). The distributions of MTHFR 677C/T genotype and allele frequencies in individuals with and without metabolic syndrome in the colorectal cancer patients group showed no definite differences (Table 3).

Clinical and biomedical variables of metabolic syndrome were individually analyzed according to 3 MTHFR 677C/T genotype in the cancer patient group, but the results showed no statistically significant differences (Table 4). Clinical and biomedical variables

Table 1. Clinical and biochemical variables for individuals with and without metabolic syndrome

\begin{tabular}{lccc}
\hline \multirow{2}{*}{ Parameter } & \multicolumn{3}{c}{ Wetabolic syndrome } \\
\cline { 2 - 4 } & $110(43.1)$ & $145(56.9)$ & - \\
\hline No. $(\%)$ & $67.2 \pm 10.7$ & $59.5 \pm 12.7$ & $<0.0001$ \\
Age (yr) & $63 / 47$ & $79 / 65$ & 0.79 \\
Gender (M/F) & $24.4 \pm 3.2$ & $21.9 \pm 2.6$ & $<0.0001$ \\
Body mass index & $144.3 \pm 70.7$ & $98.1 \pm 63.2$ & $<0.0001$ \\
Triglyceride (mg/dL) & $37.1 \pm 10.0$ & $44.0 \pm 12.5$ & $<0.0001$ \\
HDL-C (mg/dL) & $146.0 \pm 54.4$ & $113.3 \pm 37.2$ & $<0.0001$ \\
FBS (mg/dL) & $144.3 \pm 14.2$ & $135.3 \pm 15.7$ & $<0.0001$ \\
SBP (mm Hg) & $89.2 \pm 10.4$ & $84.4 \pm 10.7$ & 0.0004 \\
DBP (mm Hg) & &
\end{tabular}

$\mathrm{HDL}-\mathrm{C}$, high density lipoprotein; FBS, fasting blood sugar; SBP, systolic blood pressure; DBP, diastolic blood pressure. $\mathrm{P}<0.05$.

Table 2. Comparison of MTHFR subtypes between the control and the colorectal cancer groups

\begin{tabular}{lccc}
\hline Parameter & CC & CT & TT \\
\hline Control & $145(32.4)$ & $238(53.1)$ & $65(14.5)$ \\
Colorectal cancer & $87(34.1)$ & $134(52.6)$ & $34(13.3)$ \\
\hline
\end{tabular}

Values are presented as number (\%).

Methylenetetrahydrofolate reductase (MTHFR) 677 C > T subtypes: CC, CT and TT. in individuals were reanalyzed according to the three genotypes in the two patients group with and without metabolic syndrome.

Table 3. Distribution of methylenetetrahydrofolate reductase (MTHFR) $677 \mathrm{C} / \mathrm{T}$ genotype and allele frequencies in individuals with and without metabolic syndrome in colorectal cancer

\begin{tabular}{lcccc}
\hline Variable & $\begin{array}{c}\text { With MS } \\
(\mathrm{n}=110)\end{array}$ & $\begin{array}{c}\text { Without MS } \\
(\mathrm{n}=145)\end{array}$ & P-value & OR $(95 \% \mathrm{Cl})$ \\
\hline Genotypes & & & & \\
CC & $36(32.7)$ & $51(35.2)$ & & \\
CT & $60(54.6)$ & $74(51.0)$ & 0.678 & $0.87(0.50-1.50)$ \\
$\Pi \pi$ & $14(12.7)$ & $20(13.8)$ & 1.000 & $1.00(0.45-2.25)$ \\
Alleles & & & & \\
C & $132(60.0)$ & $176(60.7)$ & & \\
T & $88(40.0)$ & $114(39.3)$ & 0.927 & $0.97(0.67-1.39)$ \\
\hline
\end{tabular}

Values are presented as number (\%).

MS, metabolic syndrome; $\mathrm{OR}$, odds ratio; $\mathrm{Cl}$, confidence interval.

Table 4. Clinical and biomedical variables, analyzed by genotype, of metabolic syndrome in individuals with colorectal cancer $(\mathrm{n}=255)$

\begin{tabular}{lccc}
\hline Parameter & CC & CT & TT \\
\hline No. $(\%)$ & $87(34.1)$ & $134(52.6)$ & $34(13.3)$ \\
Age $(\mathrm{yr})$ & $63.41 \pm 11.60$ & $62.35 \pm 12.93$ & $63.50 \pm 13.41$ \\
Gender (M/F) & $46 / 41$ & $81 / 53$ & $16 / 18$ \\
Body mass index & $23.24 \pm 3.151$ & $23.07 \pm 3.042$ & $22.30 \pm 3.553$ \\
Triglyceride (mg/dL) & $119.8 \pm 84.68$ & $118.5 \pm 64.10$ & $111.8 \pm 52.21$ \\
HDL-C (mg/dL) & $40.12 \pm 11.92$ & $40.38 \pm 11.78$ & $43.47 \pm 12.32$ \\
FBS (mg/dL) & $128.5 \pm 50.69$ & $124.7 \pm 42.96$ & $135.7 \pm 60.21$ \\
SBP $(\mathrm{mm} \mathrm{Hg})$ & $139.5 \pm 17.12$ & $138.8 \pm 15.17$ & $140.0 \pm 14.77$ \\
DBP $(\mathrm{mm} \mathrm{Hg})$ & $86.90 \pm 11.03$ & $86.34 \pm 11.14$ & $86.47 \pm 9.497$ \\
\hline
\end{tabular}

HDL-C, high density lipoprotein; FBS, fasting blood sugar; SBP, systolic blood pressure; DBP, diastolic blood pressure.

Table 5. Clinical and biomedical variables, analyzed by genotype, in individuals with and without metabolic syndrome

\begin{tabular}{|c|c|c|c|c|c|c|c|c|c|c|c|c|}
\hline & \multicolumn{12}{|c|}{ MTHFR $677 \mathrm{C} / \mathrm{T}$ genotype } \\
\hline & \multicolumn{6}{|c|}{ With metabolic syndrome $(n=110)$} & \multicolumn{6}{|c|}{ Without metabolic syndrome $(n=145)$} \\
\hline & $\mathrm{CC}$ & CT & TT & $\mathrm{CC} / \mathrm{TT}$ & $\mathrm{CC} / \mathrm{CT}$ & Anova & $\mathrm{CC}$ & CT & $\Pi$ & $\mathrm{CC} / \mathrm{TT}$ & $\mathrm{CC} / \mathrm{CT}$ & Anova \\
\hline №. (\%) & $36(32.7)$ & $60(54.6)$ & $14(12.7)$ & & & & $51(35.2)$ & $74(51.0)$ & $20(13.8)$ & & & \\
\hline Gender (M/F) & $18 / 18$ & $38 / 22$ & $7 / 7$ & & & & $28 / 23$ & $43 / 31$ & $9 / 11$ & & & \\
\hline Age (yr) & $67.1 \pm 11.3$ & $66.9 \pm 10.8$ & $68.7 \pm 9.5$ & 0.64 & 0.90 & 0.84 & $60.7 \pm 11.1$ & $58.6 \pm 13.3$ & $59.8 \pm 14.6$ & 0.76 & 0.35 & 0.66 \\
\hline $\mathrm{BMI}\left(\mathrm{kg} / \mathrm{m}^{2}\right)$ & $24.8 \pm 3.1$ & $24.6 \pm 2.9$ & $22.6 \pm 4.0$ & 0.04 & 0.76 & 0.07 & $22.1 \pm 2.6$ & $21.7 \pm 2.5$ & $22.0 \pm 3.2$ & 0.95 & 0.49 & 0.78 \\
\hline $\mathrm{FBS}(\mathrm{mg} / \mathrm{dL})$ & $144.1 \pm 53.2$ & $142.1 \pm 50.1$ & $167.4 \pm 72.3$ & 0.21 & 0.85 & 0.28 & $117.5 \pm 46.2$ & $110.3 \pm 29.3$ & $113.6 \pm 38.1$ & 0.73 & 0.29 & 0.57 \\
\hline $\mathrm{TG}(\mathrm{mg} / \mathrm{dL})$ & $136.4 \pm 64.4$ & $150.8 \pm 76.3$ & $136.9 \pm 62.5$ & 0.97 & 0.34 & 0.57 & $108.2 \pm 95.3$ & $92.3 \pm 34.9$ & $94.3 \pm 35.6$ & 0.52 & 0.19 & 0.37 \\
\hline $\mathrm{HDL}-\mathrm{C}(\mathrm{mg} / \mathrm{dL})$ & $36.5 \pm 7.9$ & $37.8 \pm 11.6$ & $35.8 \pm 7.0$ & 0.80 & 0.58 & 0.76 & $43.5 \pm 13.9$ & $42.8 \pm 11.4$ & $50.5 \pm 12.0$ & 0.12 & 0.77 & 0.13 \\
\hline $\mathrm{SBP}(\mathrm{mm} \mathrm{Hg})$ & $143.8 \pm 13.4$ & $144.7 \pm 14.6$ & $144.3 \pm 15.5$ & 0.90 & 0.76 & 0.95 & $136.5 \pm 18.8$ & $134.1 \pm 13.9$ & $137.0 \pm 13.8$ & 0.91 & 0.41 & 0.61 \\
\hline $\mathrm{DBP}(\mathrm{mm} \mathrm{Hg})$ & $88.3 \pm 9.4$ & $90.3 \pm 11.0$ & $87.1 \pm 10.6$ & 0.70 & 0.36 & 0.48 & $85.8 \pm 12.0$ & $83.1 \pm 10.1$ & $86.0 \pm 8.8$ & 0.96 & 0.16 & 0.29 \\
\hline
\end{tabular}

BMI, body mass index; TG, triglyceride; HDL-C, high density lipoprotein; FBS, fasting blood sugar; SBP, systolic blood pressure; DBP, diastolic blood pressure. 
However, the results did not indicate any statistically significant differences (Table 5).

\section{DISCUSSION}

Metabolic syndrome is referred to as the grouping phenomenon of several metabolic risk factors, such as hypertension, diabetes, obesity, hyperlipidemia, etc., that mainly cause cardiovascular disease. Although diagnostic criteria for metabolic syndrome have been changing with different expert groups since the first guidelines of the WHO in 1998, insulin resistance and obesity have always been considered as important basic factors [3]. Metabolic syndrome, the well-known major risk factor of coronary heart disease, has now been studied for its relationship to other diseases, including cancer $[3,4]$.

In colorectal cancer studies, not only individual metabolic factor but also other related factors, such as low physical activity, abdominal obesity, etc., were evaluated for their possible relation to cancer. For example, Frezza et al. [11] insisted that being overweight was closely related to colon cancer and that the reduction of weight was important for decreasing the risk of cancer. Chang and Ulrich [12] reported that a high level of blood sugar affected the carcinogenesis of the colon. Sturmer et al. [5], in their 19-year followingup study for one healthy group, reported that being overweight and having diabetes were more closely related to colon cancer than other metabolic factors. The prevalence of metabolic syndrome in the adult population older than 50 years was reported to be approximately $45 \%$ in the United States and $16.3 \%$ in South Korea $[6,13]$. On the other hand, the prevalence of metabolic syndrome in colon cancer patients was high in our study, approaching $43 \%$.

Playing an important role for not only DNA synthesis but also DNA methylation, MTHFR takes part in the metabolism of folic acid, resulting in the formation of S-adenosyl methionine, which is mainly involved in the methylation. There are two major polymorphisms of MTHFR, 677C (alanine) $\rightarrow \mathrm{T}$ (valine) and $1298 \mathrm{~A}$ (glutamate) $\rightarrow C$ (alanine) substitutions. The alterations from these polymorphisms result in decreased efficacy of the enzyme, which might subsequently decrease DNA methylation and may be related to colorectal carcinogenesis.

The TT subtype decreases the enzyme strength by about $30 \%$ and causes hypomethylation. The TT subtype affects the blood level of folic acid, and it has been reported to increase the risk of colon cancer, especially in heavy alcohol drinkers with a low level of folic acid. The TT subtype was contrarily reported not only to decrease the risk of colon cancer but also to increase the proportion of colon cancer patients with microsatellite instability [14-16]. In this study, we couldn't find any difference of frequency for the MTHFR polymorphism between the colorectal cancer group and the healthy control group, with the colorectal cancer group showing 13.3\% TT subtype and the healthy control group showing $14.5 \%$ TT subtype. This result is similar to our previous report, showing $11.5 \%$ TT subtype in the colon cancer group and $14.4 \%$ in the control group [7].
Sporadic microsatellite instability is thought to be caused by promoter hypermethylation of the MLH1 mismatch repair gene. In the case of a low level of folic acid concentration, the TT subtype causes a large extent of hypomethylation and a consequent alteration of gene expression.

We have mentioned the TT subtype as a prognostic factor for colon cancer [7]; on the other hand, we were able to find several interesting articles about studies on the TT subtype for other fields. For example, Ellingrod et al. [10] reported that patients in the schizophrenic psychiatric patient group with the TT subtype had a 3.7fold increased risk of metabolic syndrome and suggested that this type seems to increase insulin resistance and abdominal obesity.

As mentioned earlier, on database such as PubMed, we couldn't find any reports similar to ours on the relationships of colon cancer, metabolic syndrome and MTHFR polymorphism all together, although there were several reports that individually studied only the relation between colon cancer and metabolic syndrome, and only the relation between colon cancer and MTHFR polymorphism. In this study, the distributions of MTHFR polymorphism based on the presence or absence of metabolic syndrome did not show different patterns, and even the frequencies of the $\mathrm{T}$ allele showed similar patterns. As a result, we could find no relation between metabolic syndrome and MTHFR polymorphism in colon cancer patients.

The reason we did not have any significant result, despite our large sample size compared to that of Ellingrod et al. [10], who obtained statistically significant results, is thought to be due to the particular patient group, i.e., psychiatric patients, Ellingrod et al.s. We assume that medications or other clinical factors might have contributes to their being more vulnerable to metabolic syndrome.

In order to get rid of confounding bias after surgery, we conducted this study only with patients who had been diagnosed prior to surgery as having metabolic syndrome. However, the individual factors of metabolic syndrome (hypertension, blood sugar level, cholesterol level, etc.) could have varied according to the clinical condition or the situation of the patients, and even the accuracy of medical records might be doubted in this retrospective study.

As a factor of metabolic syndrome, we adopted the BMI, instead of the abdominal circumference, because the abdominal circumference is often omitted on medical records. Especially, Korean people tend to have abdominal obesity even in the normal range of height and weight, and whether the BMI or the abdominal circumference is chosen for the diagnosis of metabolic syndrome seems to affect the results of the study. Besides, there are different criteria for the diagnosis of metabolic syndrome and agreement on criteria for a definite diagnosis has not yet been reached.

We think that a large and prospective study might be needed in order to achieve accurate and meaningful results. If there were to be a significant difference between MTHFR polymorphism in metabolic syndrome and that in colon cancer, then the comparison of survival based on polymorphism would be meaningful. Even though we have a few other reports for comparison, we have 
failed to achieve significant results in this study. Still, we believe that this study itself to be meaningful as a first report studying the relationship between MTHFR polymorphism and metabolic syndrome in colorectal cancer.

In conclusion, the results of this study revealed no relation between metabolic syndrome and MTHFR polymorphism in colorectal cancer. Although we could not elicit such a relationship, a further prospective study based on precise diagnostic criteria for metabolic syndrome may be able to establish such a relationship.

\section{CONFLICT OF INTEREST}

No potential conflict of interest relevant to this article was reported.

\section{ACKNOWLEDGEMENTS}

This work was supported by the National Research Foundation of Korean Grant funded by the Korean Government (2009-0075784).

\section{REFERENCES}

1. Ministry of Health and Welfare. Annual report of the national cancer registry in Korea 2006 [Internet]. Seoul: Ministry of Health and Welfare; 2009 [cited $2011 \mathrm{Mar}$ 20]. Available from: http://library.mw.go.kr/skyblueopen/users/bogun/PdfViewer.aspx?mastid $=63250 \&$ pdf_url=K0031255.pdf.

2. Reaven GM, Chen YD. Role of insulin in regulation of lipoprotein metabolism in diabetes. Diabetes Metab Rev 1988;4:639-52.

3. Inoue S, Zimmet P. The Asia-Pacific perspective: redefining obesity and its treatment. Sydney: Health Communications Australia Pty Ltd; 2000.

4. Lym YL, Hwang SW, Shim HJ, Oh EH, Chang YS, Cho BL. Prevalence and risk factors of the metabolic syndrome as defined by NCEP-ATP III. J Korean Acad Fam Med 2003;24:135-43.

5. Sturmer T, Buring JE, Lee IM, Gaziano JM, Glynn RJ. Metabolic abnormalities and risk for colorectal cancer in the physicians' health study. Cancer Epidemiol Biomarkers Prev 2006;15:2391-7.

6. Ju JH, Lim TK, Shin KK, Park KH, Park KC, Kim MJ, et al. Relationship between the metabolic syndrome and colorectal polyps. J Korean Acad Fam Med 2007;28:134-40.

7. Kim HS, Kang BS, Kim JW, Ahn DH, Bae SJ, Kim NG. Polymorphism of the 5,10-methylenetetrahydrofolate reductase (MTHFR) gene and microsatellite instability (MSI) in mucinous colorectal cancer. J Korean Soc Coloproctol 2008;24:329-36.

8. Lengauer C, Kinzler KW, Vogelstein B. Genetic instabilities in human cancers. Nature 1998;396:643-9.

9. Oron-Herman M, Rosenthal T, Sela BA. Hyperhomocysteinemia as a component of syndrome X. Metabolism 2003;52:1491-5.

10. Ellingrod VL, Miller DD, Taylor SF, Moline J, Holman T, Kerr J. Metabolic syndrome and insulin resistance in schizophrenia patients receiving antipsychotics genotyped for the methylenetetrahydrofolate reductase (MTHFR) 677C/T and 1298A/C variants. Schizophr Res 2008;98:47-54.

11. Frezza EE, Wachtel MS, Chiriva-Internati M. Influence of obesity on the risk of developing colon cancer. Gut 2006;55:285-91.

12. Chang CK, Ulrich CM. Hyperinsulinaemia and hyperglycaemia: possible risk factors of colorectal cancer among diabetic patients. Diabetologia 2003;46:595-607.

13. Reilly MP, Rader DJ. The metabolic syndrome: more than the sum of its parts? Circulation 2003;108:1546-51.

14. Chang SC, Lin PC, Lin JK, Yang SH, Wang HS, Li AF. Role of MTHFR polymorphisms and folate levels in different phenotypes of sporadic colorectal cancers. Int J Colorectal Dis 2007;22:483-9.

15. Shannon B, Gnanasampanthan S, Beilby J, Iacopetta B. A polymorphism in the methylenetetrahydrofolate reductase gene predisposes to colorectal cancers with microsatellite instability. Gut 2002;50:520-4.

16. Eaton AM, Sandler R, Carethers JM, Millikan RC, Galanko J, Keku TO. 5,10-methylenetetrahydrofolate reductase 677 and 1298 polymorphisms, folate intake, and microsatellite instability in colon cancer. Cancer Epidemiol Biomarkers Prev 2005;14:2023-9. 